\title{
CARACTERÍSTICAS SENSORIAIS DE LINGUIÇA ORIUNDAS DE OVELHA ALIMENTADA COM CÁRTAMO
}

Bruna Lima Chechin Catussi ${ }^{1}$; Adriana Aparecida Pinto ${ }^{2}$; Rafael Henrique de Tonissi e Buschinelli de Goes $^{3}$; Stella Maris Teobaldo Tironi ${ }^{4}$; Antonio Campanha Martinez ${ }^{5}$

1 Acadêmica do Curso de Medicina Veterinária, Universidade Estadual de Maringá Campus Umuarama

2 Professora Doutora do Departamento de Medicina Veterinária, Universidade Estadual de Maringá - Campus Umuarama

3 Professor Doutor, Curso de Zootecnia, Universidade Federal da Grande Dourados 4 Mestranda do Programa de Mestrado em Produção Sustentável e Saúde Animal, Universidade Estadual de Maringá

5 Professor Doutor Programa de Mestrado em Produção Sustentável e Saúde Animal, Universidade Estadual de Maringá, acmartinez@uem.br

Recebido em: 02/10/2017 - Aprovado em: 21/11/2017 - Publicado em: 05/12/2017 DOI: 10.18677/EnciBio_2017B11

\section{RESUMO}

A comercialização da carne de ovelhas descartes tem sido um grande obstáculo na ovinocultura, muitas vezes proporcionando prejuízo aos produtores. O presente estudo teve como objetivo avaliar sensorialmente um produto cárneo com carne de ovelhas alimentadas ou não com grão de cártamo, e diferentes proporções de toucinho suíno para observar se há ou não melhora na palatabilidade. Foram fabricados seis tipos de linguiça, a T1(sem cártamo e sem toucinho), T2 (sem cártamo e com 15\% de toucinho), T3 (sem cártamo e com 30\% de toucinho), T4 (com cártamo e sem toucinho), T5 (com cártamo e com 15\% de toucinho) e T6 (com cártamo e com $30 \%$ de toucinho). Foi observada maior preferência pela T4, T5 e T1, avaliando-se a qualidade global. A intenção de compra no mercado foi maior para T4, T6 e T3.

PALAVRAS- CHAVE: Carne, Carneiro, ovino, subproduto.

\section{SENSORIAL CHARACTERISTICS OF SAUSAGE FROM SHEEP FED WITH SAFFLOWER}

\begin{abstract}
The marketing of culled sheep meat has been a major obstacle in sheep farming, often resulting in loss to producers. The present study had the objective of sensorial evaluating a meat product with sheep meat fed or not with safflower grain, and different proportions of pork fat to observe whether or not there is improvement in palatability. Six types of sausage, T1 (without safflower and without pork fat), T2 (without safflower and with 15\% of pork fat), T3 (without safflower and with $30 \%$ of pork fat), T4 (with safflower and without pork fat), T5 (with safflower and with $15 \%$ of pork fat) and T6 (with safflower and with $30 \%$ of pork fat). A higher preference was
\end{abstract}


observed for T4, T5 and T1, and overall quality was assessed. And the intention to buy in the market was higher for T4, T6 and T3.

KEYWORDS : Ewe, meat, ovine, subproduct

\section{INTRODUÇÃO}

O consumo de carne ovina vem crescendo nos últimos anos, em 2014, o número de ovinos no país era de 17,6 milhões de cabeças (FAO, 2017), e está demasiadamente relacionado com a produção do cordeiro para a comercialização (ZEOLA, et al., 2005). Segundo Teixeira et al. (2017) aproximadamente $70 \%$ da população mundial consomem ovinos e caprinos regularmente na dieta, preferindo especialmente os cordeiros e cabritos. Entretanto, a produção de cordeiros gera ovelhas de descarte, que são ovelhas com idade avançada, que tem a eficiência reprodutiva diminuída e devem ser abatidas afim de manter a eficiência do rebanho. Na maioria das criações a reposição do rebanho materno é de $20 \%$ ao ano (BHATT, et al., 2013), e a carne desses animais é caracterizada como um produto com odor e sabor forte, sendo assim rejeitada pela maioria dos consumidores, levando a um baixo preço de comercialização e prejuízo aos produtores (TEIXEIRA et al., 2017).

Fruet et al. (2016) relataram que marmoreio na carne é o resultado do equilíbrio entre a absorção, a síntese e degradação de triglicérides e que consequentemente, as dietas que proporcionaram um maior consumo de glicose para os músculos aumentam a deposição de gordura por via intramuscular em ruminantes. Uma alternativa para o uso das carcaças provenientes de ovinos adultos é a utilização na forma de produtos cárneos, sendo esta uma estratégia viável para a indústria de alimentos (DUTRA et al., 2013), o processamento, na forma de embutidos, cozidos ou defumados, como salames, linguiças e hambúrgueres.

Os alimentos conhecidos como "funcionais" têm sido muito estudados devido aos seus efeitos benéficos para a saúde humana. Os óleos na dieta de ruminantes podem modificar as características sensoriais dos produtos, portanto além do perfil lipídico deve-se garantir a aceitabilidade do produto de animais submetidos a essas dietas. Além disso, alguns produtos e subprodutos das oleaginosas que são utilizados como fonte proteica na dieta dos ruminantes, pode substituir o farelo de soja e causar um efeito produtivo semelhante, e também a redução nos custos da dieta e aumento da rentabilidade (GOÉS et al., 2016).

O cártamo (Carthamus tinctorius L.), família Asteraceae, é uma planta oleaginosa anual, bem adaptada às condições do semiárido. As sementes do Cártamo apresentam nutrientes essenciais que compõem a dieta humana (LOPES JúNIOR et al., 2016). A composição do óleo de cártamo consiste em $90 \%$ de perfil insaturado, abundante em ácidos graxos essenciais como ácido oleico, com 20 a $30 \%$, e o ácido linolênico, com 70 a 87\% da composição (CAMPANELLA, et al., 2014).

Teve-se como proposta deste estudo desenvolver e avaliar as propriedades sensoriais de linguiças utilizando carne de ovelha de descarte, alimentadas com cártamo ou não com o intuito de verificar as características de aceitabilidade de consumo. 


\section{MATERIAL E MÉTODOS}

O estudo foi realizado no Laboratório de Criação e Reprodução Animal, localizado no campus fazenda da Universidade Estadual de Maringá, no município de Umuarama, Paraná. Foram utilizadas quatro ovelhas, com idade média de cinco anos, com escore corporal médio de 1,5, divididas em dois grupos com dois animais cada. Durante o período experimental os animais foram alojados em baias cobertas, contendo comedouros e bebedouros. Em ambos os grupos a alimentação foi fornecida em três horários (às 8h, 12h e 16h), na proporção de 3,5 \% de matéria seca em relação ao peso corporal do animal. A dieta foi balanceada conforme as recomendações do NRC (2007) e a composição bromatológica descrita na tabela 1.

No grupo 1 foi utilizado $15 \%$ de cártamo na alimentação pelo período de 60 dias, com o objetivo de alterar o perfil de ácidos graxos e no grupo 2 a dieta não teve a inclusão deste ingrediente (Tabela 2). A quantidade fornecida foi ajustada de acordo com a sobra diária, com valor de $10 \%$ e os dois grupos receberam água ad libitum.

TABELA 1. Composição bromatológica dos ingredientes das dietas experimentais.

\begin{tabular}{l|llllll} 
INGREDIENTES (\%MS) & MS & PB & EE & FDN & FDA & MM \\
\hline FARELO DE SOJA & 89,21 & 48,51 & 1,78 & 14,90 & 7,00 & 7,23 \\
FENO DE TIFTON 85 & 88,94 & 9,69 & 1,55 & 77,66 & 38,72 & 6,40 \\
GRÃO DE CÁRTAMO & 93,00 & 19,50 & 22,70 & 49,00 & 38,00 & 5,50 \\
MILHO GRÃO & 87,70 & 10,57 & 3,19 & 18,21 & 4,37 & 1,67
\end{tabular}

$\mathrm{MS}=$ Matéria Seca, $\mathrm{PB}=$ Proteína Bruta, $\mathrm{EE}=$ Extrato Etéreo, FDN = Fibra em Detergente Neutro, FDA = Fibra em Detergente Ácido, MM = Matéria Mineral.

TABELA 2. Composição percentual da dieta oferecida aos animais no período de confinamento

INGREDIENTE

GRUPO 1 (\%)

GRUPO 2 (\%)

\begin{tabular}{l|rr} 
CARTAMO GRÃO & 15,00 & 0,00 \\
MILHO GRÃO & 49,63 & 58,93 \\
MINERAL & 5,00 & 5,00 \\
SOJA FARELO & 10,37 & 16,07 \\
FENO DE TIFFTON 85 & 20,00 & 20,00
\end{tabular}

As ovelhas foram pesadas a cada 14 dias para acompanhamento do ganho de peso durante o confinamento e um dia antes do abate. Após 60 dias de confinamento os animais foram conduzidos até o abatedouro, em Iguatemi- PR, e abatidos com uso de protocolo de bem estar animal e com inspeção oficial. As carcaças foram resfriadas a $4^{\circ} \mathrm{C}$ por 24 horas em câmara fria. Após esta etapa as carcaças foram desossadas e a carne congelada para elaboração dos embutidos. A produção da linguiça foi através do uso de normas e boas práticas de fabricação de produtos de origem animal. Fazendo-se a inclusão de 0, 15 e $30 \%$ da massa final de toucinho suíno, em carne da ovelha do grupo 1 e grupo 2. A fabricação se deu pela descongelação e moagem das carnes e toucinho, separadamente. A massa foi batida e embutida em tripa suína hidratada em solução salina a $1 \%$ e assim armazenadas para avaliação sensorial.

O projeto intitulado como "Características sensoriais de linguiça oriundas de ovelha descarte alimentada com cártamo" foi aprovado pelo Comitê de Ética em pesquisa com Seres humanos sob o parecer oㅜ119708/2016. A análise sensorial foi 
realizada com 100 provadores não treinados, de ambos os sexos e de diferentes faixas etárias, que foram recrutados na Universidade Estadual de Maringá, centro de tecnologia em Umuarama- PR. Todos os provadores receberam seis amostras do produto produzidas com proporções diferentes de carne ovina e toucinho suíno (figura 1).
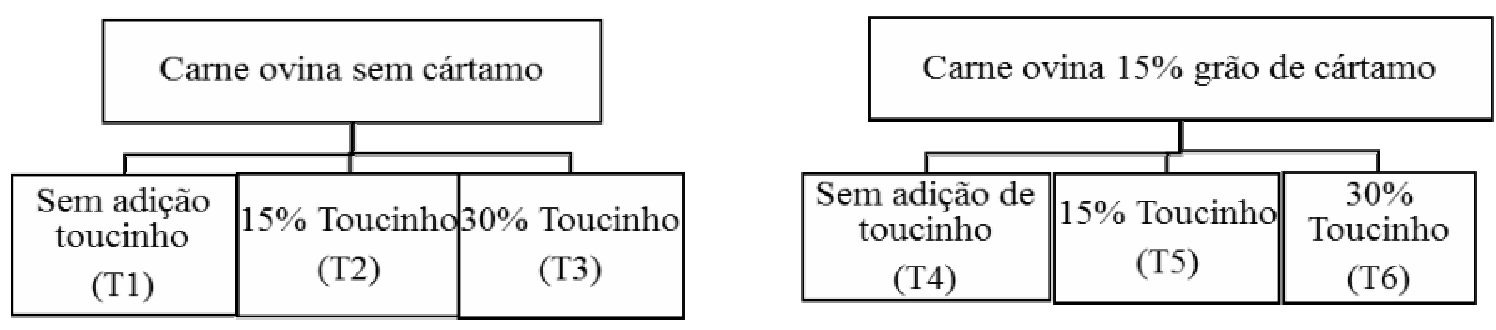

FIGURA 1. Composição das seis amostras com diferentes porcentagens de carne ovina e toucinho suíno, nomeadas de T1, T2, T3, T4, T5 e T6

As amostras de linguiça foram fritas em óleo vegetal até atingirem a temperatura de $75^{\circ} \mathrm{C}$ no interior de uma amostra. Foi cortada em aproximadamente $2 \mathrm{~cm}$ de aresta e distribuídas de forma balanceada em recipientes plásticos codificados com números aleatórios, juntamente com a ficha de avaliação (Figura 2), com termo de consentimento livre e esclarecimento (figura 3) e um copo de água. Foi aplicado o teste de aceitação utilizando escala hedônica de nove pontos, avaliando os atributos aroma, textura, suculência, sabor e qualidade global e escalada hedônica de 5 prontos, avaliando a intenção de compra das amostras. 
Nome: DATA______FICHA:

Você está recebendo uma amostra de linguiça ovina. Por favor, avalie o produto e marque na escala o quanto você gostou e desgostou das seguintes características:

\begin{tabular}{|l|}
\hline \multicolumn{1}{|c|}{ ESCALA } \\
1-Detestei \\
2- Desgostei muito \\
3- Desgostei moderadamente \\
4- Desgostei ligeiramente \\
5- Nem gostei/ nem desgostei \\
6- Gostei ligeiramente \\
7- Gostei moderadamente \\
8- Gostei muito \\
9- Adorei \\
\hline
\end{tabular}

Número da amostra:

\begin{tabular}{|c|l|}
\hline AROMA & Nota: \\
\hline TEXTURA & Nota: \\
\hline SUCULÊNCIA & Nota: \\
\hline SABOR & Nota: \\
\hline QUALIDADE GLOBAL & Nota: \\
\hline Comentários: \\
\end{tabular}

Número da amostra:

\begin{tabular}{|c|l|}
\hline AROMA & Nota: \\
\hline TEXTURA & Nota: \\
\hline SUCULÊNCIA & Nota: \\
\hline SABOR & Nota: \\
\hline QUALIDADE GLOBAL & Nota: \\
\hline Comentários: & \\
\end{tabular}

Número da amostra:

\begin{tabular}{|c|l|}
\hline AROMA & Nota: \\
\hline TEXTURA & Nota: \\
\hline SUCULÊNCIA & Nota: \\
\hline SABOR & Nota: \\
\hline QUALIDADE GLOBAL & Nota: \\
\hline Comentários: \\
\hline
\end{tabular}

INTENÇÃO DE COMPRA

$$
\begin{aligned}
& \text { 1- Certamente não compraria } \\
& \text { 2- Possivelmente não compraria } \\
& \text { 3- Talvez compraria/ talvez não compraria } \\
& \text { 4- Possivelmente compraria } \\
& \text { 5- Certamente compraria }
\end{aligned}
$$

Número da amostra:

\begin{tabular}{|c|l|}
\hline AROMA & Nota: \\
\hline TEXTURA & Nota: \\
\hline SUCULÊNCIA & Nota: \\
\hline SABOR & Nota: \\
\hline QUALIDADE GLOBAL & Nota: \\
\hline Comentários: \\
\hline
\end{tabular}

Número da amostra:

\begin{tabular}{|c|l|}
\hline AROMA & Nota: \\
\hline TEXTURA & Nota: \\
\hline SUCULÊNCIA & Nota: \\
\hline SABOR & Nota: \\
\hline QUALIDADE GLOBAL & Nota: \\
\hline Comentários:
\end{tabular}

Número da amostra:

\begin{tabular}{|c|l|}
\hline AROMA & Nota: \\
\hline TEXTURA & Nota: \\
\hline SUCULÊNCIA & Nota: \\
\hline SABOR & Nota: \\
\hline QUALIDADE GLOBAL & Nota: \\
\hline Comentários: \\
\hline
\end{tabular}

Amostra Nota

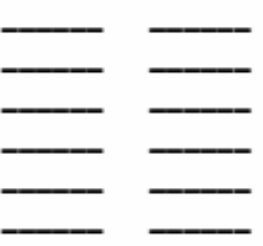

FIGURA 2. Ficha de avaliação que foi distribuída para os 100 voluntários não treinados, onde os mesmos respondiam após provar as amostras. 
O Sr. (a) está sendo convidado (a) como voluntário (a) a participar da pesquisa "Características sensoriais de linguiça oriundas de ovelha descarte alimentada com cártamo"

Nome:

Justificativa: Tendo em vista a baixa aceitabilidade para comercialização da carne de ovelha descarte, propõe-se estudos de novas tecnologias para melhorar as características

Organolépticas da carne ovina, facilitando assim a aceitação no mercado

Objetivo do projeto: Elaborar linguiça de carne ovina com influência de um tratamento nutricional com cártamo afim de aumentar concentração de ácidos graxos essenciais e a adição de gordura entremeada suína para melhorar a aceitabilidade

Procedimentos:

A análise na qual seres humanos avaliam diversos atributos de qualidade de alimentos é chamada de ANÁLISE SENSORIAL. Os procedimentos para execução da análise sensorial nesta pesquisa serão os seguintes:

- Cem provadores farão a avaliação sensorial dos produtos. Serão testadas formulações de produtos cárneos com diferentes composições. O provador deverá avaliar (olhar, cheirar, provar) os produtos e responder às perguntas solicitadas na ficha de avaliação. As amostras serão provadas individualmente, e entre as amostras, o participante receberá água filtrada para lavagem da cavidade oral e neutralização do paladar. A duração do teste para cada pessoa será aproximadamente 5 minutos. 0 nome do participante não será identificado em nenhuma publicação que possa resultar deste estudo. A sua participação poderá envolver os seguintes riscos ou desconfortos: Intolerância a alguma substância presente na formulação e náuseas brandas e vômitos brandos. Serão oferecidos água filtrada e sacos descartáveis para ser usado em qualquer momento do teste. Serão garantidos todos os cuidados necessários a sua participação de acordo com seus direitos individuais e respeito ao seu bem estar físico e psicológico

Outras informações:

- O provador pode se recusar a continuar com a avaliação sensorial a qualquer momento, sem penalização alguma e sem prejuízo ao seu cuidado. Os provadores não terão qualquer tipo de despesa em decorrência da participação nesta pesquisa. T odos os ingredientes utilizados nos produtos são realizados de acordo com as normas de boas práticas de fabricação. Em função do exposto no item anterior, não há previsão de indenização em decorrência da participação neste projeto. Em casos de restrição alimentar devido à religião ou qualquer outro tipo, deve-se avisar ao pesquisador, podendo assim abandonar a o teste sem qualquer penalização ou prejuízo. Os testes para avaliação sensorial das linguiças, nos quais os provadores experimentarão os produtos desenvolvidos e serão acompanhados pelo pesquisador proponente (Antonio C Martinez). O Provador será informado de todos os resultados, independentemente do fato destes poderem mudar o consentimento em participar da pesquisa. Este estudo é importante porque os seus resultados fornecerão dados úteis para a pesquisa. Quaisquer outros esclarecimentos poderão ser solicitados antes, durante e após a pesquisa $\mathrm{Eu}$,

RG_ CPF_ abaixo assinado, concordo em participar do estudo "Características sensoriais de linguiça oriundas de ovelha descarte alimentada com cártamo"

Tenho pleno conhecimento da justificativa, objetivos, benefícios esperados e dos procedimentos a serem executados, bem como da possibilidade de receber esclarecimentos sempre que considerar necessário. Será mantido sigilo quanto a identificação de minha pessoa e zelo a minha privacidade. Ao mesmo tempo assumo o compromisso de seguir as recomendações estabelecidas pelos pesquisadores.

Eu li e entendi todas as informações contidas neste documento

Responsável: Antonio Campanha Martinez Professor do Curso de Medicina Veterinária Contato: 44 3621-9451 ou acmartinez@uem.br

Umuarama, de de . Assinatura

FIGURA 3. Termo de consentimento livre e esclarecimento da avaliação sensorial que foi entregue e devidamente assinado aos voluntários da análise sensorial. 


\section{RESULTADOS}

Os animais (tabela 3), obtiveram como ganho de peso médio diário 232 gramas e 314 gramas, do grupo 1 e do grupo 2, respectivamente.

TABELA 3. Peso individual dos animais a cada 14 dias de confinamento e um dia antes do abate

PESO OVELHAS (KG)

\begin{tabular}{c|cccc}
\hline $\begin{array}{c}\text { DIAS DE } \\
\text { CONFINAMENTO }\end{array}$ & $10 \mathrm{SI}(\mathrm{G} 1)$ & $15 \mathrm{SI}(\mathrm{G} 1)$ & $8 \mathrm{SI}(\mathrm{G} 2)$ & $20 \mathrm{SI}(\mathrm{G} 2)$ \\
0 & 53,5 & 35 & 38,5 & 46,9 \\
14 & 62,5 & 42,5 & 44,9 & 52,8 \\
28 & 66,6 & 47 & 50,9 & 59,9 \\
42 & 64,7 & 47,6 & 51,9 & 63,6 \\
56 & 68,1 & 45 & 52,2 & 66 \\
59 & 68,8 & 47,5 & 55,7 & 67,1
\end{tabular}

O valor médio do rendimento de carcaça fria no grupo 1 foi de $40,8 \%$ e do grupo 2 de 39,9\%. Os valores de $\mathrm{pH}$ da carne de ovelha variaram entre 5,9 e 5,7, que foi mensurado 24 horas após o abate dos animais. A média das análises sensoriais utilizando-se escala hedônica de 1 a 9 , de acordo com o tipo de linguiça está descrita na tabela 4 e os gráficos individuais de acordo com cada tipo de linguiça está apresentado na figura 4.

TABELA 4. Resultado da avaliação sensorial das diferentes linguiças de ovelha descarte

OVELHA TRATADA COM 0\%

CÁRTAMO NA DIETA

\begin{tabular}{l|} 
AROMA \\
TEXTURA \\
SUCULÊNCIA \\
SABOR \\
QUALIDADE \\
GLOBAL
\end{tabular}

G $0 \%$

$(\mathrm{T} 1)$

6,09

G $15 \%(T 2)$

$\mathrm{G} 30 \%(\mathrm{~T} 3)$

6,16

6,01

6,13

6,02

6,25

6,24

6,01

6,31

6,15

6,36

6,22

6,22

6,25

6,2

OVELHA TRATADA COM 15\%

CÁRTAMO NA DIETA

$\begin{array}{ccc}\text { G } 0 \% & \text { G } 15 \% & \text { G } 30 \% \\ \text { (T4) } & \text { (T5) } & \text { (T6) } \\ 6,44 & 6,23 & 6,1 \\ 6,3 & 6,45 & 6,31 \\ 6,27 & 6,3 & 6,3 \\ 6,39 & 6,29 & 6,08 \\ 6,41 & 6,34 & 6,25\end{array}$

6,41

6,34

6,25 
T1

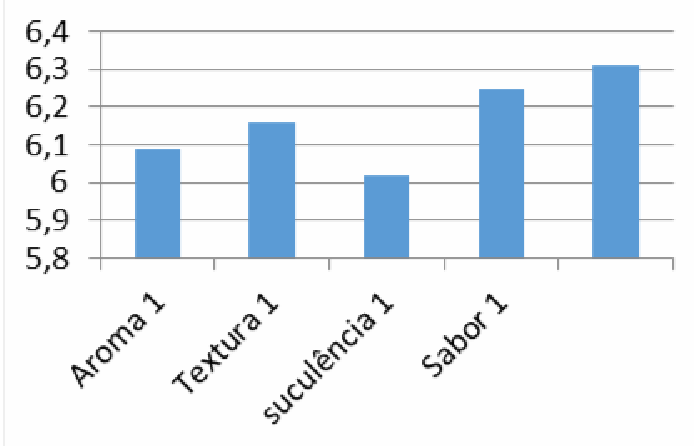

T3

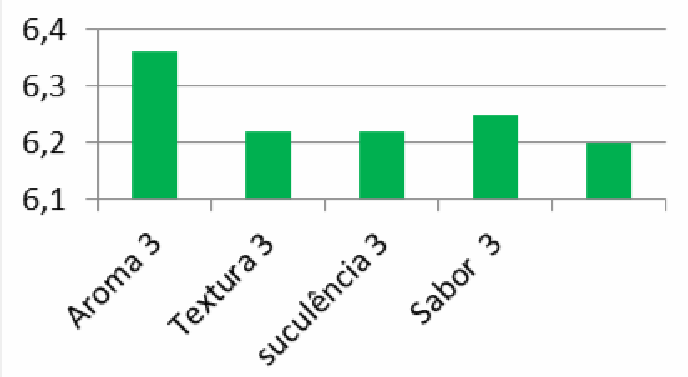

T5
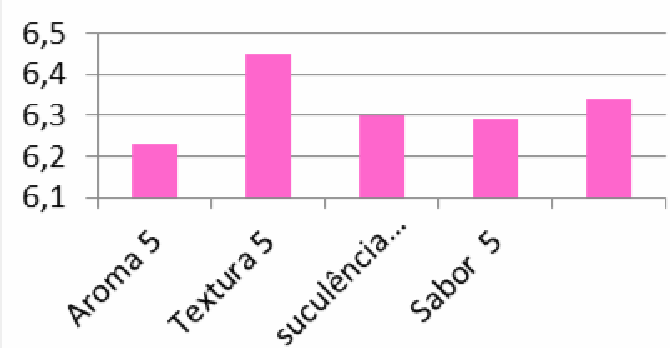

T2
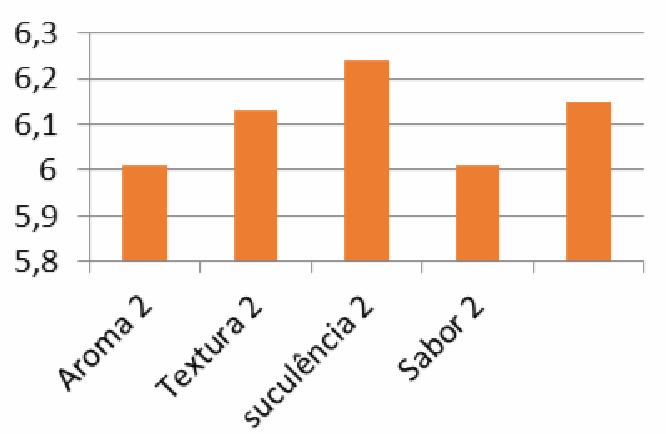

T4
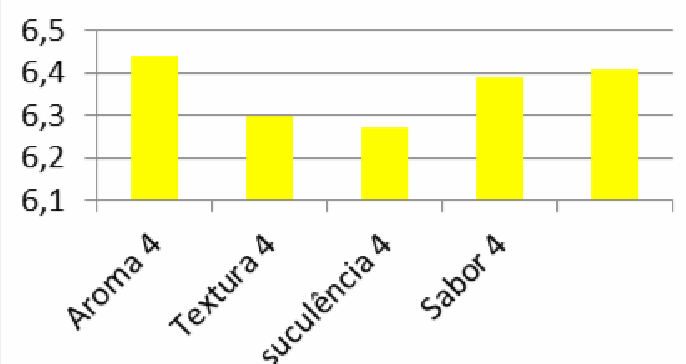

T6

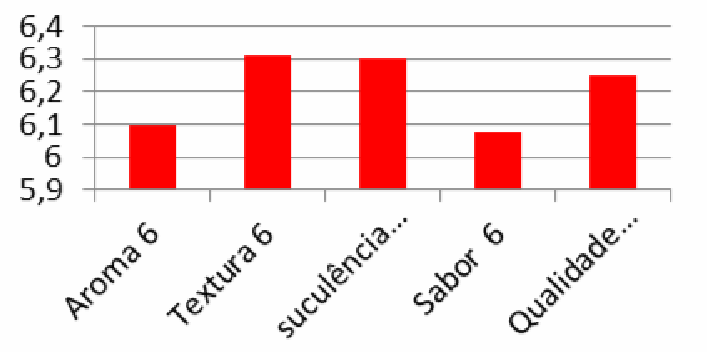

FIGURA 4. Gráfico com os resultados dos valores em escala de 1 a 9 dos atributos sensoriais (aroma, textura, suculência, sabor e qualidade global) da linguiça elaborada com carne de ovelha com diferentes proporções de toucinho suíno.

Pode-se observar com os resultados que a linguiça T4 (com cártamo e sem toucinho) obteve maior qualidade global, em segundo a T5 (com cártamo e $15 \%$ de toucinho), em seguida a T1 (sem cártamo e sem toucinho). Sobre a intenção de compra que também foi realizada, utilizando-se uma escala de 1 a 5 , os resultados na figura 5 mostram que a maior intenção de compra foi para a linguiça T4, T6, T3, T1 e por último a T2. 


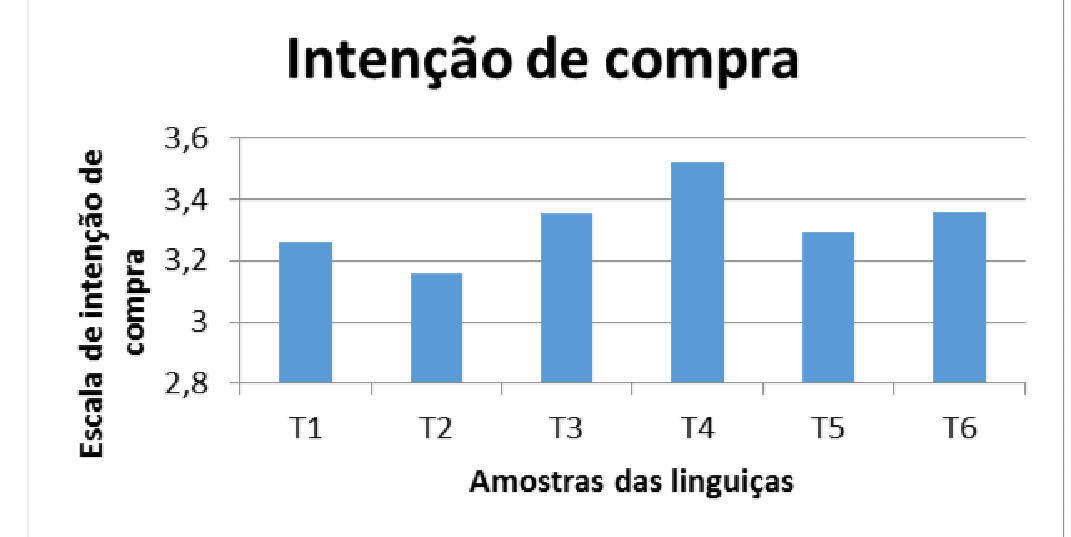

FIGURA 5. Gráfico de intenção de compra dos provadores que participaram da análise sensorial

\section{DISCUSSÃO}

Os componentes fenólicos, dos quais o Cártamo é possuidor, se apresentam como um proeminente agente antioxidante e promotores de saúde funcionais, o uso destes componentes de moléculas bioativas como aditivos alimentares são pouco estudados (MARTINS et al., 2016). Sabe-se que o objetivo do incremento do cártamo na dieta de ovelhas descarte é aumentar o valor de perfil de ácidos graxos insaturados, o ácido linoleico conjugado (CLA) por exemplo, vem sendo considerado benéfico para a saúde do homem devido as suas propriedades anticarcinogênicas $e$ metabólicas, segundo Liu e Ma (2014) alimentos com maior proporção de CLA apresentam ser benéficos para a saúde humana.

Os efeitos biológicos que causam benefícios à saúde, incluindo a capacidade antioxidante são relacionados com a inibição da carcinogênese, redução de problemas cardíacos e efeitos anti-inflamatórios (JIMÉNEZ-COLMENERO, 2013). Além disso, o óleo de cártamo é uma fonte rica em $\alpha$-tocoferois, desempenhando assim potente ação antioxidante (CAMPANELLA et al., 2014).

Leite et al. (2015) estudaram as propriedades físico-químicas, perfil de ácidos graxos e características sensoriais de salsichas de ovino e caprino fabricados com diferentes porcentagens de gordura suína, usando o grupo controle (sem adição de gordura), $10 \%$ e $30 \%$ de gordura suína. Todos os parâmetros físico-químicos foram afetados pela adição em ambos os tipos de salsichas. A adição de toucinho modificou o perfil total de ácidos graxos, uma queda significativa nas percentagens relativas dos ácidos graxos principais de ovino e caprino. A aceitabilidade foi significativamente influenciada pelo nível de gordura e pela espécie, as salsichas de cabra fabricadas com maior teor de gordura apresentaram as maiores pontuações de preferência do consumidor e em segundo a salsicha de ovinos com o teor de gordura médio.

A carne de ovinos é considerada rica em ácidos graxos saturados, pois os micro-organismos do rúmen hidrogenam extensivamente os ácidos graxos insaturados da dieta. Os ácidos graxos mais encontrados nesta espécie são: o mirístico $(2,04$ a 3,65 \%), o palmítico (20,88 a $24,22 \%)$ e o esteárico (11,89 a 15,09 $\%)$; os monoinsaturados são: o palmitoléico $(2,23$ a $2,54 \%)$ e o oléico $(31,74$ a $45,23 \%)$; e os poli-insaturados são: o linoléico (4,73 a 10,39\%), o linolênico $(0,43$ a 2,84\%) e o araquidônico (1,14 a 6,79\%) (CIRNE et al., 2016). 
No rúmen dos ovinos ocorre a hidrogenação de grande proporção dos ácidos graxos insaturados da dieta, ocorrendo maior disponibilidade de ácidos graxos saturados para absorção e deposição no tecido muscular. Contudo, quando alimentados com dietas ricas em grãos, os pequenos ruminantes acumulam grandes percentagens de ácidos graxos de cadeia longa na gordura da carne (YOUNG et al., 2003).

Além da semente de cártamo, o feno de cártamo, fornecido ad libitum, foi utilizado com sucesso como alimento exclusivo para vacas leiteiras tardias (LANDAU et al., 2004) e foi relatado para melhorar a fertilidade em ovelhas canadenses, quando comparado com o feno de pastagem de alfafa (STANFORD et al., 2001). O cártamo cultivado no estágio de brotação pode ser ensilado (WEINBERG et al., 2002) e silagem de cártamo substituída por silagem de cereais na dieta de vacas leiteiras de alto rendimento sem afetar o desempenho de laticínios (LANDAU et al., 2004).

Kott et al. (2003) avaliaram 50 cordeiros e estudaram o efeito de dietas contendo $6 \%$ de óleo de semente de cártamo (composto de $79,1 \%$ de ácido linoléico, $6,2 \%$ de palmítico, $2,1 \%$ de esteárico e 10,3\% de oléico), ou dieta controle, sem adição do óleo e concluíram que a inclusão do óleo causou efeito positivo na composição lipídica da carne, especialmente na porcentagem de CLA. Da mesma forma Huaiping Shi et al., (2015) trabalhando com inclusão de grãos de Cártamo na dieta de cabras lactantes verificaram um aumento dos componentes característicos de condição saudável no leite destes animais, principalmente na quantidade de ácidos graxos de cadeia longa.

\section{CONCLUSÃO}

A inclusão de cártamo na dieta de ovelhas descarte foi benéfica para melhorar a aceitação de carne ovina em forma de embutido, fazendo com que haja maior intenção de compra no mercado.

\section{AGRADECIMENTO}

Ao $\mathrm{CnPQ}$ pelo pagamento de Bolsa de Iniciação Científica à primeira autora

\section{REFERÊNCIAS}

BHATT, R.S.; SOREN, M.N.; SAHOO, A.; KARIM, S.A. Level and period of realimentation to assess improvement in body condition and carcass quality in cull ewes. Tropical Animal Health and Production, v. 45, p.167-176. 2013. Disponível em: <https://doi.org/10.1007/s11250-012-0188-z> doi: $10.1007 / \mathrm{s} 11250-012-0188-z$

CAMPANELLA, L. C. A.; SILVA, A. C.; FREYGANG, J.; DAL MAGRO, D. D. Efeito da suplementação de óleo de cártamo sobre o peso corporal, perfil lipídico, glicídico e antioxidante de ratos wistar induzidos a obesidade. Revista de Ciência Farmacêuticas Básica e Aplicada, v.35, n.1, p.141-147, 2014. Disponível em: http://serv-bib.fcfar.unesp.br/seer/index.php/Cien_Farm/article/view/2921/1526

CIRNE, L. G. A.; SOBRINHO, A. G. S.; ALMEIDA, F. A.; SANTANA, V. T.; ENDO, V.; ZEOLA, N. B. L.; ALMEIDA, A. K. Características da carcaça de cordeiros alimentados com dietas contendo feno de amoreira. Semina: Ciências Agrárias, v. 37, n. 4, p. 2737-2748, 2016. doi: 10.5433/1679-0359.2016v37n4Supl1p2737 
DUTRA, M.P.; PALHARES, P.C.; SILVA, J.R.O.; EZEQUIEL, I.P.; RAMOS, A.L.S.; PEREZ, J.R.O.; RAMOS, E.M. Technological and quality characteristics of cooked ham-type pâté elaborated with sheep meat. Small Ruminant Research, v.115, p. 56-61, 2013. Disponível em: <https://doi.org/10.1016/j.smallrumres.2013.08.007>. doi: 10.1016/j.smallrumres.2013.08.007

FAO. FAOSTAT Producti on live animals. Disponível em: < http://faostat3.fao.org/download/Q/QA/E>. Acesso em: 22 out. 2017.

FRUET, A. P. B.; STEFANELLO, F. S.; JUNIOR, A. G. R.; DE SOUZA, A. N. M.; TONETTO, C. J.; NORNBERG, J. L. Wholegrains in the finishing of culled ewes in pasture or feedlot: Performance, carcass characteristics and meat quality. Meat

Science. v.113, p. 97-103. 2016. Disponível em: <https://doi.org/10.1016/j.meatsci.2015.11.018> doi: 10.1016/j.meatsci.2015.11.018

GOES, R. H. T. B.; CARNEIRO, M. M. Y.; BRABES, K. C. S.; LANA, R. P. Coprodutos de Crambe (Crambe abyssinica Hoechst) na alimentação de ruminantes. Archivos de Zootecnia, v.65, p.7-16, 2016. Disponível em: <http://dx.doi.org/10.21071/az.v65i249.450> doi: $10.21071 / a z . v 65 i 249.450$

HUAIPING SHI, J. L.; WEI, Z.; HEJUN, S. Using safflower supplementation to improve the fatty acid profile in milk of dairy goat. Small Ruminant Research, v.127, p.68-73, 2015. Disponível em: <https://doi.org/10.1016/j.smallrumres.2015.04.001> doi: 10.1016/j.smallrumres.2015.04.001

JIMÉNEZ-COLMENERO, F. Potential applications of multiple emulsions in the development of healthy and functional foods. Food Research International, v. 52, p, 64-74, 2013. Disponível em: <https://doi.org/10.1016/j.foodres.2013.02.040> doi: 10.1016/j.foodres.2013.02.040

KOTT, R. W.; HATFIELD, P. G.; BERGMAN, J. W.; FLYNN, C. R.; VAN AGONER, H.; BOLES, J. A. Feedlot performance, carcass composition, and muscle and fat CLA concentrations of lambs fed diets supplemented with safflower seeds. Small Ruminant Research. V.49, P. 11-17, 2003. Disponível em: <https://doi.org/10.1016/S0921-4488(03)00052-X>. doi: 10.1016/S0921-4488(03)00052-X

LANDAU, S.; FRIEDMAN, S.; DEVASH, L.; MABJEESH, S.J. Polyethylene glycol, determined by near-infrared reflectance spectroscopy, as a marker of fecal output in goats. Journal of Agricultural and Food Chemistry, v.50, p.1374-1378. 2002 DOI:10.1021/jf011346b

LEITE, A.; RODRIGUES, S; PEREIRA, E.; PAULOS, K.; OLIVEIRA, A. F.; LORENZO, J. M.; TEIXEIRA, A. Physicochemical properties, fatty acid profile and sensory characteristics of sheep and goat meat sausages manufactured with different pork fat levels. Meat Science, v. 105. p. 114-120. 2015.

Disponível em: <https://doi.org/10.1016/j.meatsci.2015.03.015> doi: 10.1016/j.meatsci.2015.03.015 
LIU, J.; MA, D. W. L. The role of $n-3$ polyunsaturated fatty acids in the prevention and treatment of breast cancer. Nutrients, v.6, p. 5184-5223, 2014. Disponível em: <https://doi.org/10.3390/nu6115184> doi: $10.3390 /$ nu6115184

LOPES JÚNIOR, C.A.; OLIVEIRA, S.R.; MAZZAFERA, P.; ARRUDA,M.A.Z.; Expanding the information about the influence of cadmium on the metabolism of sunflowers: Evaluation of total, bioavailable, and bioaccessible content and metallobiomolecules in sunflower seeds. Environmental and Experimental Botany, v.125, p. 87-97, 2016 https://doi.org/10.1016/j.envexpbot.2016.02.003

MARTINS, N.; RORIZ, C.L.; MORALES, P.; BARROS, L.; FERREIRA, I.C.F.R. Food colorants: Challenges, opportunities and current desires of agroindustries to ensure consumer expectations and regulatory practices. Trends in Food Science \& Technology, v. 52, p.1-15, 2016. Disponível em: $<$ https://doi.org/10.1016/j.tifs.2016.03.009> doi: 10.1016/j.tifs.2016.03.009

NCR - NATIONAL RESEARCH COUNCIL - . Nutrient requirement of small ruminants: sheep, goats, cervids, and new world camelids. 6th ed. Washington, DC: National Academy Press, p. 383, 2007.

STANFORD, K.; WALLINS, G.L.; LEES, B.M.; MUNDEL, H.H. Feeding value of immature safflower forage for dry ewes. Canadian Journal of Animal Science, v. 81, p 289-292, 2001. https://doi.org/10.4141/A00-090

TEIXEIRA, A.; FERNANDES, A.; PEREIRA, E.; MANUEL, A., RODRIGUES, S. Effect of salting and ripening on the physicochemical and sensory quality of goat and sheep cured legs. Meat Science, v.134, p.163-169, 2017. Disponível em: <https://doi.org/10.1016/j.meatsci.2017.08.002>. doi: 10.1016/j.meatsci.2017.08.002

WEINBERG, Z.G.; ASHBELL, G.; HEN, Y.; LESHEM, Y.; LANDAU, S., BRUCKENTAL, I. A note on ensiling safflower forage. Grass and Forage Science, v. 57, p 184 - 187. 2002. Disponível: https://doi.org/10.1046/j.13652494.2002.00314.x doi: 10.1046/j.1365-2494.2002.00314.x

YOUNG, O.A.; LANE, G.A.; PRIOLO, A. Pastoral and species flavour in lambs raised on pasture, lucerne or maize. Journal of the Science of Food and Agriculture, v.83, n.2, p.93-104, 2003. Disponível em: <https://doi.org/ 10.1002/jsfa.1282>. doi: 10.1002/jsfa. 1282

ZEOLA, N. M. B. L.; SOBRINHO, A. G. S.; SOUZA, P. A.; SOUZA, H. B. A.; PELICANO, E. R. L.; LEONEL, F. R.; LIMA, T. M. Avaliação da injeção de cloreto de cálcio nos parâmetros qualitativos da carne de ovelha. Revista Brasileira de Agrociência. v. 11, n. 3 , p. 361-364. 2005. Disponível em: http://www2.ufpel.edu.br/faem/agrociencia/v11n3/artigo17.pdf 